\title{
Synthesis of 5-(alkylthio or arylthio)-3H-1,2-dithiol-3-one derivatives
}

\author{
Alejandro M. Fracaroli, Jeronimo Kreiker, Rita H. de Rossi, and Alejandro M. Granados" \\ Instituto de Investigaciones en Físico Química de Córdoba (INFIQC), Departamento de \\ Química Orgánica, Facultad de Ciencias Químicas, Universidad Nacional de Córdoba, Ciudad \\ Universitaria, 5000 Córdoba, Argentina \\ E-mail: ritah@mail.fcq.unc.edu.ar, ale@mail.fcq.unc.edu.ar
}

\section{Dedicated to Prof. Joan Bosch on the occasion of his $60^{\text {th }}$ Birthday}

\begin{abstract}
5-(R,S)-3H-1,2-dithiol-3-one derivatives with $\mathrm{R}$ =butyl, ethyl, phenyl, cyclopentyl, octyl, $\mathrm{p}$ chlorophenyl, p-bromophenyl and p-methoxyphenyl were prepared in moderate to good yield from the reaction of the corresponding 5-(R,S)-3H-1,2-dithiol-3-thione with $\mathrm{Hg}\left(\mathrm{CH}_{3} \mathrm{COO}\right)_{2}$ in glacial acetic acid.
\end{abstract}

Keywords: $3 H$-1,2-Dithiol-3-one, $3 H$-1,2-dithiol-3-thione, synthesis

\section{Introduction}

The heterocyclic pseudoaromatic compounds $3 H$-1,2-dithiole-3-thiones $\mathbf{1}$ have been known for several years. ${ }^{1}$

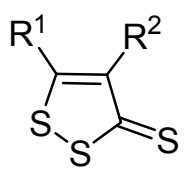

A wide variety of alkyl and aryl derivatives have been synthesized and a great number of these compounds display biological activity and have industrial applications. For instance, Oltipraz (4-methyl-5-pyrazinyl-3H-1,2-dithiole-3-thione) was originally used as an antischistosomal agent due to its remarkable activity against Schistosoma mansoni. ${ }^{2}$ In addition, studies have demonstrated that Oltipraz inhibits HIV-1 (AIDS) ${ }^{3}$ virus replication by irreversibly binding to the viral reverse transcriptase enzyme. This compound has also shown 
chemoprotective activity against a great variety of carcinogens and investigations are in progress to determine its probable use as a chemoprotective agent. ${ }^{4-6}$ Other derivatives like 4-aryl-5chloro-3H-1,2-dithiole-3-thiones have proven to be fungitoxic ${ }^{7}$ and they have also been used as insecticides. ${ }^{8}$ We reported that several derivatives of $\mathbf{2}$ have interesting activity as antimycotic agents ${ }^{9}$ and that their solubility increases in the presence of $\beta$-cyclodextrin. ${ }^{10}$ Besides, it has been claimed $^{11}$ that the antifungicidal activity of derivatives $\mathbf{1}$ increases when the thiocarbonyl group is replaced by a carbonyl group.

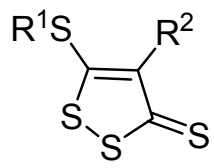

2

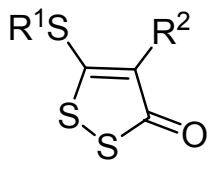

3

We therefore undertook a study in order to develop a method to synthesize $\mathbf{3}$ and the results are presented here.

\section{Results and Discussion}

Several methods have been used to exchange the sulfur by oxygen in 3H-1,2-dithiol-3-thione derivatives 1. Some examples include treatment with $\mathrm{KMnO}_{4}$ in acidic acetone, ${ }^{12}$ or the reaction with $\mathrm{Cl}_{2} / \mathrm{CCl}_{4}$ and then with water. ${ }^{13}$ More recently Torroba has reported that $\mathrm{EtO}_{2} \mathrm{C}-\mathrm{CNO}^{-}$in THF gives excellent yields of the oxidation products in compounds that have the same nucleus as 1. ${ }^{14}$ The presence of an extra sulfur atom in compound 2 limits the use of oxidants because the alkyl or arylthio function can be easily oxidized to sulfoxide. ${ }^{15}$ The most frequently used method utilizes mercury acetate in acetic acid as reagent. ${ }^{16}$ We show here that derivatives $\mathbf{2 a - i}$ can be converted into the corresponding $\mathbf{3}$ in moderate to good yields using these reagents (see Table 1). Although no other product could be isolated and the substrate was completely consumed, the low yield probably results from ring opening reactions giving rise to an untreatable mixture of products.

Attempts to do the reaction using $\mathrm{KMnO}_{4}$ in acidic acetone gave only decomposition products while $\mathrm{Cl}_{2} / \mathrm{CCl}_{4}$ led to about $5 \%$ yield of the expected product starting from compound 2a. It was reported that treatment with $\mathrm{Bi}\left(\mathrm{NO}_{3}\right)_{3}$ gave good results in the transformation of thioamides into amides ${ }^{17}$ but this methodology applied to 2 a yielded only $30 \%$ of $\mathbf{3 a}$. 
Table 1. Yield of compounds 3a-i obtained

\begin{tabular}{cccc}
\hline $\mathbf{2}$ & $\mathrm{R}^{1}$ & $\mathrm{R}^{2}$ & $\mathbf{3} \%^{\mathrm{a}}$ \\
\hline $\mathrm{a}$ & butyl & $\mathrm{H}$ & 70 \\
$\mathrm{~b}$ & ethyl & $\mathrm{Cl}$ & 43 \\
$\mathrm{c}$ & phenyl & $\mathrm{H}$ & 26 \\
$\mathrm{~d}$ & ethyl & $\mathrm{H}$ & 30 \\
$\mathrm{e}$ & cyclopentyl & $\mathrm{H}$ & 57 \\
$\mathrm{f}$ & octyl & $\mathrm{H}$ & 54 \\
$\mathrm{~g}$ & p-chlorophenyl & $\mathrm{H}$ & 18 \\
h & p-bromophenyl & $\mathrm{H}$ & 21 \\
$\mathrm{i}$ & p-methoxyphenyl & $\mathrm{H}$ & 43 \\
\hline
\end{tabular}

${ }^{\mathrm{a}}$ Isolated yield

\section{Experimental Section}

General Procedures. ${ }^{1} \mathrm{H}$ and ${ }^{13} \mathrm{C}$ NMR were recorded on a Bruker $200 \mathrm{MHz}$ instrument using TMS as an internal standard. Chemical shifts are reported in $\delta(\mathrm{ppm})$. IR spectra were recorded on a Nicolet 5SXC spectrometer using $\mathrm{KBr}$ pellets. Reagents used were obtained from commercial suppliers or synthesized as indicated below. The high resolution mass spectrometry analyses were carried out in the University of California, Riverside.

\section{General synthetic procedures}

Synthesis of compounds 2. The compounds 2 were prepared according to the method described in the literature. ${ }^{18}$ A typical run is the following: $15.7 \mathrm{mmol}$ of $\mathrm{P}_{2} \mathrm{~S}_{5}, 9.7 \mathrm{mmol}_{\text {of }} \mathrm{S}_{8}, 0.032 \mathrm{mmol}$ of 2-mercaptobenzothiazole (MBT) and $0.016 \mathrm{mmol}$ of $\mathrm{ZnO}$ were loaded into a three-necked round bottomed flask and xylene $(40 \mathrm{~mL})$ was added. The mixture was then boiled under $\mathrm{N}_{2}$ and the corresponding dithiolmalonate $(15.7 \mathrm{mmol})$, dissolved in $40 \mathrm{~mL}$ of xylene, was added dropwise for 30 minutes under continuous stirring. The reaction was boiled for an additional 1.5 hour. The color changed from light yellow to dark reddish brown. Then, the crude reaction mixture was filtered and the solvent evaporated. The dried extract was purified by column chromatography over silica gel 70-230 mesh and $\mathrm{CH}_{2} \mathrm{Cl}_{2} /$ hexane 50:50 was used as eluent.

Synthesis of compounds 3.1 mmole of 2 dissolved in $30 \mathrm{~mL}$ of acetone, freshly distilled over $\mathrm{KMnO}_{4}$, was loaded into a dry three-necked round bottomed flask under a $\mathrm{N}_{2}$ atmosphere and the solution warmed to $50^{\circ} \mathrm{C}$. Afterwards 2 mmoles of $\left(\mathrm{CH}_{3} \mathrm{COO}\right)_{2} \mathrm{Hg}$ dissolved in $2 \mathrm{~mL}$ of glacial acetic acid were slowly added and the solution was stirred additionally for $30 \mathrm{~min}$. A saturated solution of $\mathrm{NaHCO}_{3}$ was added to stop the reaction and after filtering it, it was extracted with $\mathrm{CHCl}_{3}$. The product was purified by column chromatography on silica gel eluted with solvents of 
increasing polarity from hexane to hexane $/ \mathrm{CH}_{2} \mathrm{Cl}_{2} 50 \% \mathrm{v} / \mathrm{v}$. Compounds $\mathbf{3 b}, \mathbf{3 h}$ and $\mathbf{3 i}$ were crystalline solids and were purified further by recrystallization in hexane (3b and $\mathbf{3 i})$ or $\mathrm{CHCl}_{3}$ (3h).

\section{Compound characterization}

Most of the compounds are obtained as a yellow or orange oil with strong and unpleasant odor. Compound $\mathbf{3 b}, \mathbf{3 h}$ and $\mathbf{3 i}$ are crystalline solids ( $\mathbf{3 b}$ and $\mathbf{3 h}$ yellow and $\mathbf{3 i}$ orange). A typical UV-Vis spectrum is shown in Figure 1 where the spectrum of $\mathbf{2}$ is also shown for comparison.

5-Butylthio-3H-1,2-dithiol-3-one (3a). ${ }^{13} \mathrm{C} \mathrm{NMR}\left(50 \mathrm{MHz}, \mathrm{CDCl}_{3}\right)$ : 13.42; 21.77; 30.86; 34.57; 116.46; 171.03; 192.09. ${ }^{1} \mathrm{H}$ NMR $\left(\mathrm{CDCl}_{3}\right): 0.89$ (t, $\left.6.94 \mathrm{~Hz}, 3 \mathrm{H}\right) ; 1.47(\mathrm{~m}, 2 \mathrm{H}) ; 1.72$ (m, 2H); $3.08(\mathrm{t}, 7.3 \mathrm{~Hz}, 2 \mathrm{H}) ; 6.37(\mathrm{~s}, 1 \mathrm{H}) . \mathrm{IR} \mathrm{cm} \mathrm{cm}^{-1}(\mathrm{KBr}): 617.2 ; 794.9 ; 939.7 ; 1110.8 ; 1229.3 ; 1512.2$; $1636.6 ; 2868.0 ; 2927.2 ; 2960,1 ; 3078.6$. HRMS calculated for $\mathrm{C}_{7} \mathrm{H}_{10} \mathrm{OS}_{3} 205.9894$, found 205.9896.

4-Chloro-5-ethylthio-3H-1,2-dithiol-3-one (3b). ${ }^{13} \mathrm{C} \mathrm{NMR}\left(50 \mathrm{MHz}, \mathrm{CDCl}_{3}\right)$ : 14.27; 26.23; 116.76; 161.76; 184.11. ${ }^{1} \mathrm{H}$ NMR $\left(\mathrm{CDCl}_{3}\right): 1.49$ (t, $\left.7.66 \mathrm{~Hz}, 3 \mathrm{H}\right) ; 3.20$ (c, $\left.7.66 \mathrm{~Hz}, 2 \mathrm{H}\right) . \mathrm{IR} \mathrm{cm}^{-1}$ (KBr): 663.3; 801.5; 841.0; 979.2; 1183.2; 1492.5; 1643.9; 2927.2; 2979.9. HRMS calculated for $\mathrm{C}_{5} \mathrm{H}_{5} \mathrm{ClOS}_{3} 211.9191$, found $211.9189 . \mathrm{mp} 98.4-99.3^{\circ} \mathrm{C}\left(\mathrm{lit}^{19} 104^{\circ} \mathrm{C}\right)$

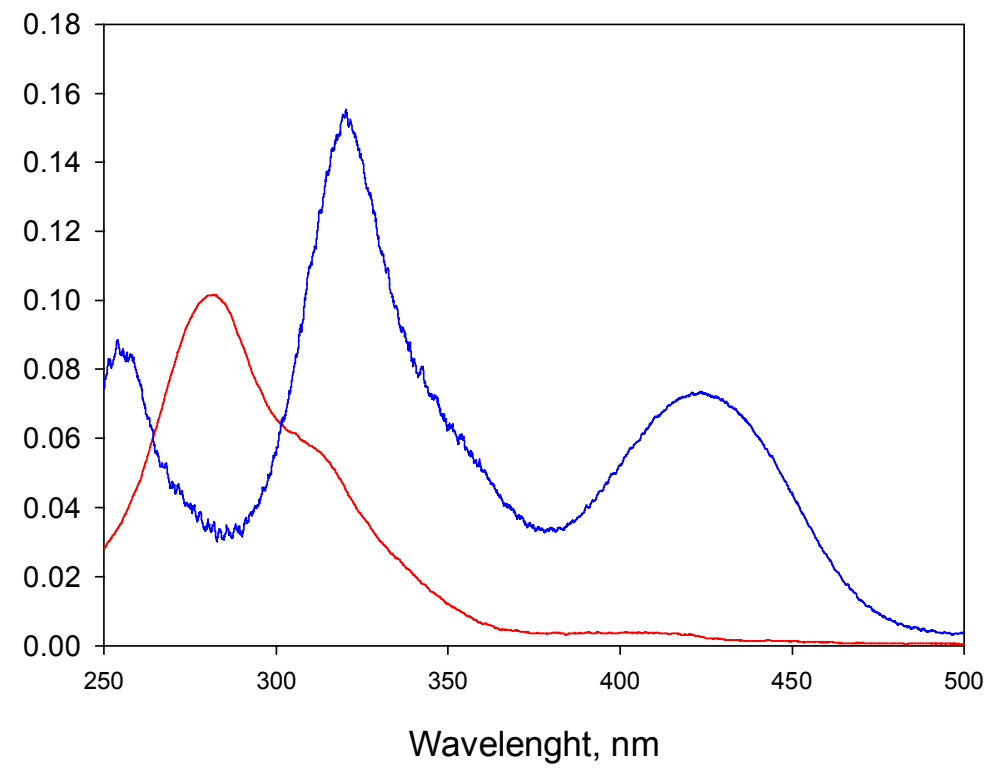

Figure 1. Uv-vis spectrum of 5-butylthio-3H-1,2-dithiol-3-ona ( - ) and 5-butylthio-3H-1,2dithiol-3-thione (- $1.2 \times 10^{-5} \mathrm{M}$ in hexane.

5-Phenylthio-3H-1,2-dithiol-3-one (3c). ${ }^{13} \mathrm{C} \mathrm{NMR}\left(50 \mathrm{MHz}, \mathrm{CDCl}_{3}\right)$ : 116.22, 126.97, 129.96, 131.25, 135.67, 171.79, 191.90. ${ }^{1} \mathrm{H}$ NMR $\left(\mathrm{CDCl}_{3}\right): 6.39(\mathrm{~s}, 1 \mathrm{H}) ; 7.47-7.62(\mathrm{~m}, 5 \mathrm{H}) . \mathrm{IR} \mathrm{cm}{ }^{-1}$ (KBr): 620.8, 691.2, 753.3, 807.1, 947.8, 1026.4, 1121.6, 1237.4, 1444.3, 1477.5, 1506.7, 1643.0, 1670.0, 2363.0, 3050.0. HRMS calculated for $\mathrm{C}_{9} \mathrm{H}_{6} \mathrm{OS}_{3} 225.9581$, found 225.9589. 
5-Ethylthio-3H-1,2-dithiol-3-one (3d): ${ }^{13} \mathbf{C}$ NMR (50 $\left.\mathbf{M H z}, \mathbf{C D C l}_{3}\right)$. 14.01, 29.05, 116.48, 170.65, 192.03. ${ }^{1} \mathrm{H}$ NMR $\left(\mathrm{CDCl}_{3}\right): 1.44$ (t, $\left.7.32 \mathrm{~Hz}, 3 \mathrm{H}\right), 3.13$ (C, $\left.7.32 \mathrm{~Hz}, 2 \mathrm{H}\right), 6.40$ (s, 1H). IR $\mathrm{cm}^{-1}(\mathrm{KBr}):$ 613.2, 799.4, 934.8, 1108.3, 1379.1, 1506.0, 1654.1, 2360.8, 1915.1, 2970.2. HRMS calculated for $\mathrm{C}_{5} \mathrm{H}_{6} \mathrm{OS}_{3} 177.9581$ found 177.9584 .

5-Cyclopentylthio-3H-1,2-dithiol-3-one (3e). ${ }^{13} \mathrm{C} \mathrm{NMR}\left(50 \mathrm{MHz}, \mathrm{CDCl}_{3}\right)$ : 24.77, 33.66, 47.54; 116.70, 171.17, 192.03. ${ }^{1} \mathrm{H}$ NMR $\left(\mathrm{CDCl}_{3}\right): 1.72(\mathrm{~m}), 2.17(\mathrm{~m}), 3.75(\mathrm{~m}), 6.37(\mathrm{~s}) . \mathrm{IR} \mathrm{cm}{ }^{-1}(\mathrm{KBr})$ : 611.4, 790.5, 930.6, 1105.8, 1226.4, 1448.3, 1506.7, 1646.8, 1658.5, 2861.2, 2954.6, 3075.3. HRMS calculated for $\mathrm{C}_{8} \mathrm{H}_{10} \mathrm{OS}_{3} 217.9894$ found 217.9890 .

5-Octylthio-3H-1,2-dithiol-3-one (3f). ${ }^{13} \mathrm{C} \mathrm{NMR}$ (50 MHz, $\mathrm{CDCl}_{3}$ ): 13.96, 22.50, 28.51, 28.81, 28.92, 31.59, 34.71, 34.85, 116.30, 170.57, 191.92. ${ }^{1} \mathrm{H}$ NMR: 0.86 (t, 3H), 1.26 (m, 10H), 1.72 $(\mathrm{m}, 2 \mathrm{H}), 3.07(\mathrm{t}, 7.68 \mathrm{~Hz}, 2 \mathrm{H}), 6.37(\mathrm{~s}, 1 \mathrm{H}) . \mathrm{IR} \mathrm{cm} \mathrm{cm}^{-1}(\mathrm{KBr}): 494.7,619.2,724.3,790.5$, 938.4, $113.6,1226.4,1382.1,1452.2,1506.7,1670.2$, 2366.9, 2853.4, 2923.5, 2958.5, 3059.7. HRMS calculated for $\mathrm{C}_{11} \mathrm{H}_{18} \mathrm{OS}_{3} 262.0520$ found 262.0523 .

5-(4-Chlorophenylthio)-3H-1,2-dithiol-3-one (3g). ${ }^{13} \mathrm{C}$ NMR (50 MHz, CDCl3): 116.81, 125.51, 130.34, 136.91, 138.10, 170.71, 191.84. ${ }^{1} \mathrm{H}$ NMR $\left(\mathrm{CDCl}_{3}\right): 6.42(\mathrm{~s}, 1 \mathrm{H}), 7.43-7.60$ (dd, 4H). IR cm ${ }^{-1}(\mathrm{KBr}): 615.3,755.5,802.2,825.5,950.1,1020.1,1090.2,1113.6,1230.3,1269.3$, $1393.8,1475.5,1506.7,1576.7,1646.8,1666.3,2359.1,2767.8,2849.6,2923.5,3071.4$. HRMS calculated for $\mathrm{C}_{9} \mathrm{H}_{5} \mathrm{ClOS}_{3} 259.9191$ found 259.9195 .

5-(4-Bromophenylthio)-3H-1,2-dithiol-3-one (3h). ${ }^{13} \mathrm{C} \mathrm{NMR}$ (50 $\left.\mathrm{MHz}, \mathrm{CDCl}_{3}\right)$ : 117.02 , 126.40, 132.23, 133.38, 137.05, 170.47, 191.93. ${ }^{1} \mathrm{H} \mathrm{NMR}\left(\mathrm{CDCl}_{3}\right): 6.43(\mathrm{~s}, 1 \mathrm{H}), 7.56(\mathrm{~m}, 4 \mathrm{H})$. IR $\mathrm{cm}^{-1}$ (KBr): 532.4, 605.5, 728.8, 798.2, 815.7, 1006.4, 1068.7, 1111.5, 1384.0, 1465.7, 1504.6, 1559.1, 1651.5, 1660.5, 2851.4, 2925.3. mp 56.9-57.9 ${ }^{\circ} \mathrm{C}$

5-(4-Metoxyphenylthio)-3H-1,2-dithiol-3-one (3i). ${ }^{13} \mathrm{C}$ NMR (50 MHz, CDCl3): 55.55, 115.25, 115.60, 117.08, 137.97, 162.33, 173.79, 192.25. ${ }^{1} \mathrm{H}$ NMR (CDCl3): 3.87 (s, 3H), 6.39 (s, 1H), $6.98(\mathrm{~m}, 2 \mathrm{H}), 7.57$ (m, 2H). IR cm ${ }^{-1}(\mathrm{KBr}):$ 530.8, 606.5, 794.1, 830.3, 948.8, 1021.2, 1106.8, $1169.3,1248.3,1291.1,1409.2,1457.1,1491.9,1584.0,1650.5,1660.1,2834.7,2930.1$, 3068.35. mp 60.9-61.9 ${ }^{\circ} \mathrm{C}$. HRMS calculated for $\mathrm{C}_{10} \mathrm{H}_{8} \mathrm{O}_{2} \mathrm{~S}_{3} 255,9686$ found 255,9681.

\section{Acknowledgements}

This work was supported in part by the Consejo Nacional de Investigaciones Científicas y Técnicas (CONICET), Agencia Nacional de Ciencia y Tecnología (FONCYT), and Secretaría de Ciencia y Tecnología (Universidad Nacional de Córdoba). AMF is a grateful recipient of a fellowship from CONICET. 


\section{References and Footnotes}

1. Landis, P. S. Chem. Rev. 1965, 65, 237. (b) Pedersen, C. Th. Adv. Heterocyclic Chem. 1982, 31, 63 and Sulfur Rep. 1995, 16, 173.

2. (a) Barreau, M.; Cotrel, C.; Jeanmart, C. Ger. Offen. Patent 2,627,211; Chem. Abst. 1977, 86, 121373. (b) Barreau, M.; Cotrel, C.; Jeanmart, C. Ger. Offen. Patent 2,705,641; Chem. Abst. 1977, 87, 152171.

3. (a) Prochaska, H. J.; Yeh, Y.; Baron, P.; Polsky, B. Proc. Natl. Acad. Sci. USA 1993, 90, 3953. (b) Prochaska, H. J.; Rubinson, L.; Yeh, Y.; Baron, P.; Polsky, B. Molecular Pharmacology 1991, 45, 916. (c) Prochaska, H. J.; Fernandes, C. L.; Pantoja, R. M.; Chavan, S. J. Biochem. Biophys. Res. Comm. 1996, 221, 548.

4. Kensler, T. W.; Groopman, J. D.; Eaton, D. L.; Curphey, T. J.; Roebuck, B. D. Carcinogenesis 1992, 13, 95. (b) Prestera, T.; Talalay, P. Proc. Natl. Acad. Sci. USA 1995, 92, 8965. (c) Begleiter, A.; Leith, M. K.; Curphey, T. J. Brit. J. Cancer 1996, 74, S9-S14. (d) Begleiter, A.; Leith, M. K.; Curphey, T. J.; Doherty, G. P. Oncol. Res. 1997, 9, 371. (e) Kim, W.; Gates, K. S. Chem. Res. Toxicol. 1997, 10, 296. (f) Doherty, G. P.; Leith, M. K.; Wang, X.; Curphey, T. J.; Begleiter, A. Brit. J. Cancer 1998, 77, 1241. (g) Munday, R.; Munday C. M. Carcinogenesis, 2004, 25, 1721.

5. Kensler, T. W.; Groopman, J. D.; Eaton, D. L.; Curphey, T. J.; Roebuck, B. D. Carcinogenesis 1992, 13, 95.

6. Prestera, T.; Talalay, P. Proc. Natl. Acad. Sci. USA 1995, 92, 8965

7. (a) Hagen, H.; Fleig, H. Ger. Offen. Patent 2,460,783; Chem. Abst. 1976, 85, 123899. (b) Bader, J.; Gaetzi, K. Ger. Offen. Patent 1, 278,701; Chem. Abst. 1969, 70, 115147.

8. Misra, P.; Misra, S.; Mohapatra, R.; Mittra, A. J. Indian Chem. Soc. 1979, 61, 404.

9. Giannini, F. A; Aimar, M. L.; Sortino, M.; Gomez, R.; Sturniollo, A.; Juarez, A.; Zacchino, S.; de Rossi, R. H.; Enriz, R. D. Il Farmaco, 2004, 59, 245.

10. Zoloff Michoff, M. E.; Granados, A. M.; de Rossi, R. H. Arkivoc 2005, (xii), 47.

11. Misra, P.; Misra, S.; Mohapatra, R.; Mittra A. J. Indian Chem. Soc. 1979, LVI, 404.

12. Clesse, F.; Pradere, J-P.; Quiniou, H.; Bull. Soc. Chim. France 1973, 586.

13. (a) Jurgen, G.; Boberg, F. Liebigs Ann. Chemie, 1978, 387. (b) Spindt, R.; Stevens, D.; Baldwin, W. E. J. Am. Chem. Soc. 1951, 73, 3693. (c) Quiniou, H.; Lozac'h, N. Bull. Chem. Soc. France 1963, 210, 1167.

14. Marcos, C.; Polo, C., Rakitin, O.; Rees, C.; Torroba, T. Chem. Commun 1997, 879.

15. Rossi, L. I.; de Rossi, R. H. Applied Catalysis, 2004, 267(1-2), 267.

16. Fesun, I. N.; Timoshenko, V. M. ; Chernega, A. N. ; Shermolovich, Yu. G. Russian J. Org. Chem. 2006, 42, 124.

17. Mohammadpoor-Baltork, I.; Khodaei, M. M; Nikoofar, K. Tetrahedron Lett. 2003, 44, 591.

18. Aimar, M. L.; Kreiker, J.; de Rossi. R. H. Tetrahedron Lett., 2002, 43, 1947.

19. Bader, J. Helv. Chim. Acta 1968, 51, 1409. 